\title{
Dexmedetomidine Attenuates Hippocampal Damage and Improves Cognition by Up-Regulating Glyoxalase1 in APP/PS1 Mice
}

\section{Chunyan Guo}

the Affiliated Hospital of Inner Mongolia Medical University

\section{Lei Zhang}

the Affiliated Hospital of Inner Mongolia Medical University

\section{Yaoxing Gao}

the Affiliated Hospital of Inner Mongolia Medical University

Junzhi Sun

the Affiliated Hospital of Inner Mongolia Medical University

\section{Lingling Fan}

the Affiliated Hospital of Inner Mongolia Medical University

\section{Yuguang Bai}

the Affiliated Hospital of Inner Mongolia Medical University

Jing Zhang

the Affiliated Hospital of Inner Mongolia Medical University

\section{Gaowa Naren}

Tongliao Hospital

\section{Jiwen Yang}

the Affiliated Hospital of Inner Mongolia Medical University

Libiao Li ( $\square$ llibiao0320@163.com )

the Affiliated Hospital of Inner Mongolia Medical University

\section{Research Article}

Keywords: Dexmedetomidine, Alzheimer's disease, GLO-1, methylglyoxal, apoptosis

Posted Date: May 25th, 2021

DOI: https://doi.org/10.21203/rs.3.rs-512431/v1

License: (c) (1) This work is licensed under a Creative Commons Attribution 4.0 International License.

Read Full License 


\section{Abstract}

Background: Dexmedetomidine (DEX), an a2-adrenoceptor agonist, has been reported to possess neuroprotective effects against postoperative cognitive impairment. GLO-1 plays a key role in the pathogenesis of Alzheimer's disease (AD). Here, the primary goal was to assess whether DEX affect GLO1 and protect cognition impairment in APP/PS1 transgenic mice.

Methods: After DEX was intraperitoneally injected in APP/PS1 mice, behavior was tested by Water Maze to illustrate whether DEX treatment has a significantly positive effect on ameliorating the cognition deficits in AD. We assessed the effect of DEX on the expression of GLO-1 and the production of other oxidative stress factors by ELISA and Western blot. To determine whether DEX play roles in the A $\beta$ induced neuron apoptosis, flow cytometry was used.

Results: DEX treatment significantly ameliorated cognition deficits in APP/PS1 mice. DEX increased GLO1 expression and decreased MG activity in the hippocampus. In addition, DEX increased activity of SOD, GSH and reduced the activity of MDA. In vitro, DEX could protect the neuron apoptosis induced by $A \beta$. GLO-1 inhibitor could block the protective role of DEX.

Conclusion: Taken together, our findings suggest that DEX prevents progression of AD-like pathology through upregulating GLO-1.

\section{Introduction}

Alzheimer's disease (AD) is characterized by a gradual deterioration of cognitive function compared to healthy patients, which may be associated with a significant reduction in brain volume in $A D$ patients $(1,2)$. The causes of atrophy are synaptic degeneration and neuronal death, especially in the hippocampus, which plays a role in memory and spatial orientation (3). Age is the highest risk factor for $A D$ and peoples over 85 have a 50 per cent risk the disease $(4,5)$.

A number of evidences show that oxidative stress plays an important role in AD pathogenesis $(6,7)$. The brain is particularly vulnerable to oxidative damage because it has:1) a large number of easily oxidized polyunsaturated fatty acids, 2) a high level of reactive oxygen species (ROS) and catalyst iron,3) a relatively lack of antioxidant capacity $(8,9)$. Oxidative stress is thought to be a common factor for $A D(10)$. Glyoxalase-1 (Glo1) is a key driver in oxidative stress mediators.Glo1 is a cytosolic protein that forms the acetaldehyde enzyme system together with acetaldehydease 2 and glutathione(11). The major function of the system is the detoxification of active diethyls, especially methylglyoxal (MG)(12). MG is a highly active metabolite of diethylglycolysis. MG is degraded by the acetaldehydease system, which is an efficient enzymatic detoxification system in which Glo1 is a rate-limiting enzyme(13). It was reported that Glo1 overexpression protected lifespan reduction, neurostructural damage and neurofunctional damage. Glo1 down-regulation also occurred in oxidative stress-induced anxiety and memory impairment in rats(14). 
Dexmedetomidine (DEX) is a highly selective a2-adrenoceptor agonist used as an off-label medication for pediatric sedation and analgesia(15). DEX was reported to exhibit neuroprotective efficacy in several brain injury models (16). In neonatal rats, DEX $(20 \mu \mathrm{g} / \mathrm{kg})$ injection enhances spatial learning and memory in 36 days old (17). In mice, DEX ameliorated sleep deprivation-induced deterioration of shortterm memory and spatial learning ability(18). In the study, we investigated whether DEX can counteract cognition impairment and regulate Glo1 expression in APP/PS1 transgenic AD mouse model.

\section{Materials And Methods}

\subsection{Materials}

DEX was purchased from Selleck. Annexin V-FITC Apoptosis Detection Kit and MTT were purchased from Sigma-Aldrich. GLO-1 inhibitor was purchased from MCE. GLO-1 and $\beta$-actin primary antibodies were purchased from Abcam. SOD, GSH, MDA, GLO-1 and MG ELISA kits were purchased from Nanjing Jiancheng Biotechnology.

\subsection{Animal}

6 months old male APP/PS1 mice were purchased from Beijing Zhongke Zisheng Biotechnology Co., Ltd. 6 months old male C57/BL6 mice were set as Con group. These mice were feed with free access to water and food. The procedures followed were assessed and approved by the Committee on the Ethics of the affiliated hospital of Inner Mongolia medical university.

\subsection{DEX administration}

When mice were feed to 7 months old, APP/PS1 transgenic mice were randomly assigned to two groups: (1) APP/PS1 group were treated with $0.9 \%$ saline by intraperitoneal injection once daily for 28 days $(n=$ 10). (2) DEX group were treated with $20 \mathrm{ug} / \mathrm{kg} D E X$ by intraperitoneal injection once daily for 28 days $(\mathrm{n}=$ 10). 7 months old C57/BL6 male mice served as Con group for APP/PS1 transgenic mice $(n=10)$.

\subsection{Water maze}

Water maze assess cognitive function by training mice to use spatial cues in a room to navigate to a hidden escape platform. The water maze apparatus was composed of a circular pool $(100 \mathrm{~cm}$ diameter, $50 \mathrm{~cm}$ high) filled with water and divided into four imaginary quadrants. At the beginning of the swim, one mouse which faced the wall was placed into the pool from the quadrant which is opposite to the platform and ended once the animal had found the platform; if the mouse had not found the platform within $60 \mathrm{sec}$, it was guided there by hand. This process will continue 5 days. On day 6 , each mouse was placed into the pool to find the platform in $60 \mathrm{~s}$. A video camera connected with a trail analysis system was set above the center of the pool.

\subsection{Western blotting}


Brain hippocampal tissues were homogenized and Western blotting performed using rabbit polyclonal antibodies GLO-1 (Abcam, 1:500) and $\beta$-actin (Abcam, 1:2000). Blots were scanned and measured using LiCor Odyssey software.

\subsection{ELISA test}

GLO-1, MG, SOD, GSH and MDA in hippocampus homogenates and cells were determined using ELISA kit.

\subsection{Cell culture}

SH-SY5Y human neuroblastoma cell line was used for in vitro experiments. The cell line was purchased from ATCC. Cells were cultured in Dulbecco's Modified Eagle's Medium supplemented with $10 \%$ fetal calf serum. When indicated, the cells were treated with $25 \mathrm{uM} \mathrm{A \beta 25-35}$ for $24 \mathrm{~h}$.

\subsection{MTT assay}

MTT[3-(4,5-dimethylthiazol-2-yl)-2,5-diphenyltetrazolium bromide] method is based on the ability of mitochondrial dehydrogenase in living cells to cut the tetrazolium ring of yellowish MTT and form dark blue methyl nitrogen crystals. MTT was operated according to the instructions.

\subsection{Flow cytometry}

Cell death was assessed using an annexin V-FITC and PI apoptosis detection kit. Cells were harvested using trypsin and incubated for $5 \mathrm{~min}$ at room temperature in the dark with Annexin V-FITC and propidium iodide (PI) according to the manufacturer's instructions. The fluorescence of FITC and PI was analyzed by flow cytometry.

\subsection{Statistical evaluation}

One-way ANOVA was used. A value of $p<0.05$ was considered significant.

\section{Results}

\subsection{DEX prevents cognitive decline in APP/PS1 mice}

The cognition was detected by Water Maze. APP/PS1 mice shows increased escape latency time and distance compared to Con group. Administration of $20 \mathrm{ug} / \mathrm{kg}$ DEX for 28 days prevented the cognitive decline. As shown in Fig. 1A-B, DEX significantly decreased the escape latency time and distance in APP/PS1 mice.

\subsection{DEX increases Glo-1 activity and decreases MG activity in hippocampus and serum}

To find out whether DEX regulate Glo-1 system, we measured the Glo-1 activity and the protein expression. As shown in Fig. 2A-C, much less expression of Glo1 in hippocampus and serum was observed in APP/PS1 mice than in the Con group. DEX treatment could significantly increase the Glo-1 
activity and the protein expression. Subsequently, we examined MG levels in serum. Normally, MG can be catalyzed into lactic acid by Glo-1. MG itself has cytotoxic effects. As shown in Fig. 2D, the level of serum MG was significantly higher in APP/PS1 mice than in the Con group. After DEX treatment, the serum MG level was decreased. These data suggested that DEX could regulate Glo-1 system.

\subsection{DEX increases SOD, GSH and decreases MDA levels in APP/PS1 mice}

APP/PS1 mice exhibited decreased levels of SOD, GSH and increased level of MDA in hippocampus and serum compared with Con group. DEX treatment significantly reversed these changes (Fig. 3).

\subsection{To determine the role of GLO-1 downregulation in A $\beta$ induced cell viability decline}

$A \beta 25-35$ is the fragment obtained by hydrolysis of $A \beta$ protein in vitro. It does not exist in the body, but its neurotoxicity is almost equal to that of endogenous $A \beta$. We first developed a SY5Y cell model of $A \beta 25-$ 35 damage. Exposure SY $5 Y$ cells to $A \beta$ for $24 \mathrm{~h}$ caused cell viability decline(Fig. $4 \mathrm{~A}$ ). Then we detected the GLO-1 and MG levels in the cells. The results showed that $A \beta$ exposure to SY $5 Y$ cells decreased GLO-1 and increased MG levels (Fig. 4B-C). It suggested that GLO-1 downregulation is involved in A $\beta$ induced cell viability decline. To further characterize the function of GLO-1 in A $\beta$ induced cell viability decline, we used GLO-1 inhibition and MG to assess their effects on cell viability. MTT analysis showed that both GLO-1 inhibition and MG decreased cell viability (Fig. 4D). These results identified that GLO-1 participated in $A \beta$ induced $S Y 5 Y$ cell viability decline.

\subsection{DEX inhibited cell apoptosis through upregulating GLO-1}

Cell apoptosis was increased after incubation with AB. GLO-1 inhibitor also increased the cell apoptosis. Treatment with DEX at $1 \mathrm{uM}$ significantly decreased the cell apoptotic rate. The protective effect of DEX was blocked in the cell model of $A \beta$ damage when incubating GLO-1 inhibitor for DEX 2 hours in advance (Fig. 5A-B).Furthermore, DEX increased the GLO-1 level and GLO-1 inhibitor can inhibit the up-regulation of GLO1 by DEX(Fig. 5 C).

\subsection{DEX treatment increases SOD, GSH and decreases MDA levels in neurons exposed to $A \beta$}

The levels of SOD and GSH in A $\beta$ group were markedly lower than those in Con group. After DEX treatment for $24 \mathrm{~h}$, the levels of SOD and GSH were significantly higher than those in the A $\beta$ group. Meanwhile, the MDA level in A $\beta$ group was markedly higher than that in Con group. After DEX treatment for $24 \mathrm{~h}$, the MDA level was significantly lower than that in the A $\beta$ group (Fig. 6).

\section{Discussions}


$A D$ pathological features are mainly progressive exacerbation of cognitive impairment (19). AD is mostly diagnosed later when neuronal cell death is irreversible. Furthermore, there are no therapeutic drugs that can effectively halt AD progress (20). The APP/PS1 mouse model has been widely used to study cognitive deficits related to $A D(21)$. Here, we report that (i) DEX alleviates cognitive impairment in APP/PS1 mice. (ii) DEX blocks Aß-induced neuron apoptosis. (iii) DEX plays it role through regulating GLO-1.

In this study, we propose DEX regulate multiple pathological changes that are important in AD and suggest repositioning the DEX to regulate lots of aspects of the AD. Dex is an efficacious, safe drug used to sedate patients in the ICU or during procedural sedation(22). We found that DEX enhance AD cognitive deficits which upregulating GLO-1 to block MG-mediated neurotoxicity. DEX can also regulate the activities of oxidative stress related enzymes such as SOD, GSH and MDA.

Oxidative stress has been proposed as a common factor for AD. Among all oxidative stress mediators, GLO-1 plays an important role(23). GLO-1 is an enzyme that detoxifies MG. MG is an effective precursor of advanced glycation end products and is considered to be a key factor in neuronal injury $(24,25)$. DEX administration halts AD progression in APP/PS1 mice, it may be through regulating GLO-1. In the study, we observed that DEX reversed the changes of GLO-1 and MG in APP/PS1 mice model. Interaction between GLO-1 and MG might provide A 3 -related cell death pathology in $A D$ and also provide a potential drug target for the disease.

Then, we identified the role of GLO-1 in cell model mediated by $A \beta$ injury. We selected $A \beta 25-35$ to injure SHSY $5 Y$ cells. A $25-35$ is a fragment between $25-35$ A $\beta$ peptide which showing neurotoxic activity in cultured cells(26). SH-SY5Y cell is a human neuroblastoma cells with sustainable cell morphology and biochemistry of mature human neurons(27). In the SH-SY5Y cell model injuried by $A \beta 25-35$, we found $A \beta$ led to cell viability decline. A $\beta$ also decreased GLO-1 and MG levels. GLO-1inhibition and MG decreased cell viability. It suggested that GLO-1 down-regulation is involved in $A \beta$ induced cell viability decline. DEX significantly decreased the cell apoptotic rate. GLO-1 inhibitor could block the protective effect of DEX. DEX increased the GLO-1 level in the cell model too.

It has been reported that bilateral microinjection of MG is sufficient to induce changes in anxiety-like behavior(28). The function of GLO-1, the AGE/RAGE signaling pathway and the generation of reactive oxygen species were all abnormal in MG-treated HUVECs(29). Considerable evidence and our work, it has shown that the expression of Glo1 can be up-regulated by EDX. Although the mechanisms still remain unclear, the role of GLO-1has been increasingly acknowledged.

In summary, we presented evidence that 28 days administration of EDX relieved oxidative stress, elevated the protein level of GLO-1 to reduce MG accumulation and ameliorated memory deficits in APP/PS1 transgenic mice. All of these benefits were related to reduce neuron apoptosis in AD process. Although the molecule mechanism requires further exploration, DEX may serve as a potential anti dementia drug. 


\section{Declarations}

Ethics approval and consent to participateđAll experimental protocols were approved by Ethics Committee of the Affiliated Hospital of Inner Mongolia Medical University. All methods were carried out in accordance with relevant guidelines and regulations. All methods are reported in accordance with ARRIVE guidelines for the reporting of animal experiments.

Consent for publication: Not applicable.

Availability of data and materials: The datasets used and/or analysed during the current study available from the corresponding author on reasonable request.

Competing interests: There were no competing interests.

Funding: Science and Technology Million Project of Inner Mongolia Medical University (YKD2015KJBW022).

Authors' contributions: Chunyan Guo, Lei Zhang and Yaoxing Gao performed the experiment. Junzhi Sun, Lingling Fan and Yuguang Bai contributed to data analysis. Jing Zhang and Gaowa Naren helped perform the data analyses and wrote the original manuscript. Jiwen Yang and Libiao Li contributed to the conception of the study and approved the final manuscript.

Acknowledgements: Not applicable.

\section{References}

1. Bondi MW, Edmonds EC, Salmon DP.Alzheimer's Disease: Past, Present, and Future.J Int Neuropsychol Soc. 2017 Oct;23(9-10):818-831.

2. Toepper M.Dissociating Normal Aging from Alzheimer's Disease: A View from Cognitive Neuroscience.J Alzheimers Dis. 2017;57(2):331-352.

3. Mu Y, Gage FH. Adult hippocampal neurogenesis and its role in Alzheimer's disease.Mol Neurodegener. 2011 Dec 22;6:85.

4. A Armstrong R.Risk factors for Alzheimer's disease. Folia Neuropathol. 2019;57(2):87-105.

5. Meunier B. Age and Alzheimer's Disease. Nutrients. 2016 Jun 16;8(6):372.

6. Tönnies E, Trushina E. Oxidative Stress, Synaptic Dysfunction, and Alzheimer's Disease.J Alzheimers Dis. 2017;57(4):1105-1121.

7. Cheignon C, Tomas M, Bonnefont-Rousselot D, Faller P, Hureau C, Collin F.Oxidative stress and the amyloid beta peptide in Alzheimer's disease.Redox Biol. 2018 Apr;14:450-464

8. Freitas HR, Ferreira GDC, Trevenzoli IH, Oliveira KJ, de Melo Reis RA. Fatty Acids, Antioxidants and Physical Activity in Brain Aging. Nutrients. 2017 Nov 20;9(11):1263.

9. Wang Y, Zhang SX, Gozal D.Reactive oxygen species and the brain in sleep apnea. Respir Physiol Neurobiol. 2010 Dec 31;174(3):307-16. 
10. Mecocci P, Boccardi V, Cecchetti R, Bastiani P, Scamosci M, Ruggiero C, Baroni M.A Long Journey into Aging, Brain Aging, and Alzheimer's Disease Following the Oxidative Stress Tracks. J Alzheimers Dis. 2018;62(3):1319-1335.

11. Antognelli C, Mezzasoma L, Mearini E, Talesa VN.Glyoxalase 1-419C $>A$ variant is associated with oxidative stress: implications in prostate cancer progression.PLoS One. 2013 Sep 10;8(9):e74014.

12. Nigro C, Leone A, Raciti GA, Longo M, Mirra P, Formisano P, Beguinot F, Miele C.MethylglyoxalGlyoxalase 1 Balance: The Root of Vascular Damage.Int J Mol Sci. 2017 Jan 18;18(1):188.

13. Stratmann B, Goldstein B, Thornalley PJ, Rabbani N, Tschoepe D.Intracellular Accumulation of Methylglyoxal by Glyoxalase 1 Knock Down Alters Collagen Homoeostasis in L6 Myoblasts. Int J Mol Sci. 2017 Feb 23;18(3):480.

14. Patki G, Solanki N, Atrooz F, Allam F, Salim S.Depression, anxiety-like behavior and memory impairment are associated with increased oxidative stress and inflammation in a rat model of social stress.Brain Res. 2013 Nov 20;1539:73-86

15. Olutoyin A Olutoye, Chris D Glover, John W Diefenderfer, Michael McGilberry, Matthew M Wyatt, Deidre R Larrier, Ellen M Friedman, Mehernoor F Watcha. The effect of intraoperative dexmedetomidine on postoperative analgesia and sedation in pediatric patients undergoing tonsillectomy and adenoidectomy.Anesth Analg. 2010 Aug;111(2):490-5.

16. Mason KP, Kelhoffer ER, Prescilla R, Mehta M, Root JC, Young VJ, Robinson F, Veselis RA.Feasibility of measuring memory response to increasing dexmedetomidine sedation in children. $\mathrm{Br} \mathrm{J}$ Anaesth. 2017 Feb;118(2):254-263.

17. Yahan Zhang, Qiushi Gao, Ziyi Wu, Hang Xue, Bo Liu, Ping Zhao.Dexmedetomidine Promotes Hippocampal Neurogenesis and Improves Spatial Learning and Memory in Neonatal Rats. Drug Des Devel Ther. 2019 Dec 31;13:4439-4449.

18. Lakkyong Hwang, II-Gyu Ko, Jun-Jang Jin, Sang-Hoon Kim, Chang-Ju Kim 1 , Boksoon Chang, Jeong Ho Rho, Eun-Jin Moon, Jae-Woo Yi. Dexmedetomidine ameliorates memory impairment in sleepdeprived mice. Anim Cells Syst (Seoul). 2019 Nov 16;23(6):371-379.

19. Serý O, Povová J, Míšek I, Pešák L, Janout V.Molecular mechanisms of neuropathological changes in Alzheimer's disease: a review.Folia Neuropathol. 2013;51(1):1-9.

20. Selkoe D, Mandelkow E, Holtzman D.Deciphering Alzheimer disease.Cold Spring Harb Perspect Med. 2012 Jan;2(1):a011460.

21. Zheng H, Zhou Q, Du Y, Li C, Xu P, Lin L, Xiao J, Gao H. The hypothalamus as the primary brain region of metabolic abnormalities in APP/PS1 transgenic mouse model of Alzheimer's disease. Biochim Biophys Acta Mol Basis Dis. 2018 Jan;1864(1):263-273.

22. Duan X, Coburn M, Rossaint R, Sanders RD, Waesberghe JV, Kowark A.Efficacy of perioperative dexmedetomidine on postoperative delirium: systematic review and meta-analysis with trial sequential analysis of randomised controlled trials. Br J Anaesth. 2018 Aug; 121(2):384-397.

23. Tseng YT, Tsai YH, Fülöp F, Chang FR, Lo YC.2-lodo-4'-Methoxychalcone Attenuates MethylglyoxalInduced Neurotoxicity by Activation of GLP-1 Receptor and Enhancement of Neurotrophic Signal, 
Antioxidant Defense and Glyoxalase Pathway.Molecules. 2019 Jun 16;24(12):2249.

24. Suantawee $T$, Thilavech $\mathrm{T}$, Cheng $\mathrm{H}$, Adisakwattana S.Cyanidin Attenuates Methylglyoxal -Induced Oxidative Stress and Apoptosis in INS-1 Pancreatic $\beta$-Cells by Increasing Glyoxalase- 1 Activity.Nutrients. 2020 May 6;12(5):1319.

25. Haddad M, Perrotte M, Khedher MRB, Demongin C, Lepage A, Fülöp T, Ramassamy C.Methylglyoxal and Glyoxal as Potential Peripheral Markers for $\mathrm{MCI}$ Diagnosis and Their Effects on the Expression of Neurotrophic, Inflammatory and Neurodegenerative Factors in Neurons and in Neuronal DerivedExtracellular Vesicles.Int J Mol Sci. 2019 Oct 3;20(19):4906.

26. Hu L, Zhang R, Yuan Q, Gao Y, Yang MQ, Zhang C, Huang J, Sun Y, Yang W, Yang JY, Min ZL, Cheng J, Deng $Y$, Hu X.The emerging role of microRNA-4487/6845-3p in Alzheimer's disease pathologies is induced by Aß25-35 triggered in SH-SY5Y cell.BMC Syst Biol. 2018 Dec 14;12(Suppl 7):119.

27. Omar SH, Scott CJ, Hamlin AS, Obied HK.Olive Biophenols Reduces Alzheimer's Pathology in SHSY5Y Cells and APPswe Mice.Int J Mol Sci. 2018 Dec 30;20(1):125.

28. McMurray KM, Du X, Brownlee M, Palmer AA.Neuronal overexpression of Glo1 or amygdalar microinjection of methylglyoxal is sufficient to regulate anxiety-like behavior in mice.Behav Brain Res. 2016 Mar 15;301:119-23.

29. Wang Z, Zhang J, Chen L, Li J, Zhang H, Guo X.Glycine Suppresses AGE/RAGE Signaling Pathway and Subsequent Oxidative Stress by Restoring Glo1 Function in the Aorta of Diabetic Rats and in HUVECs.Oxid Med Cell Longev. 2019 Mar 3;2019:4628962.

\section{Figures}


Fig1

A

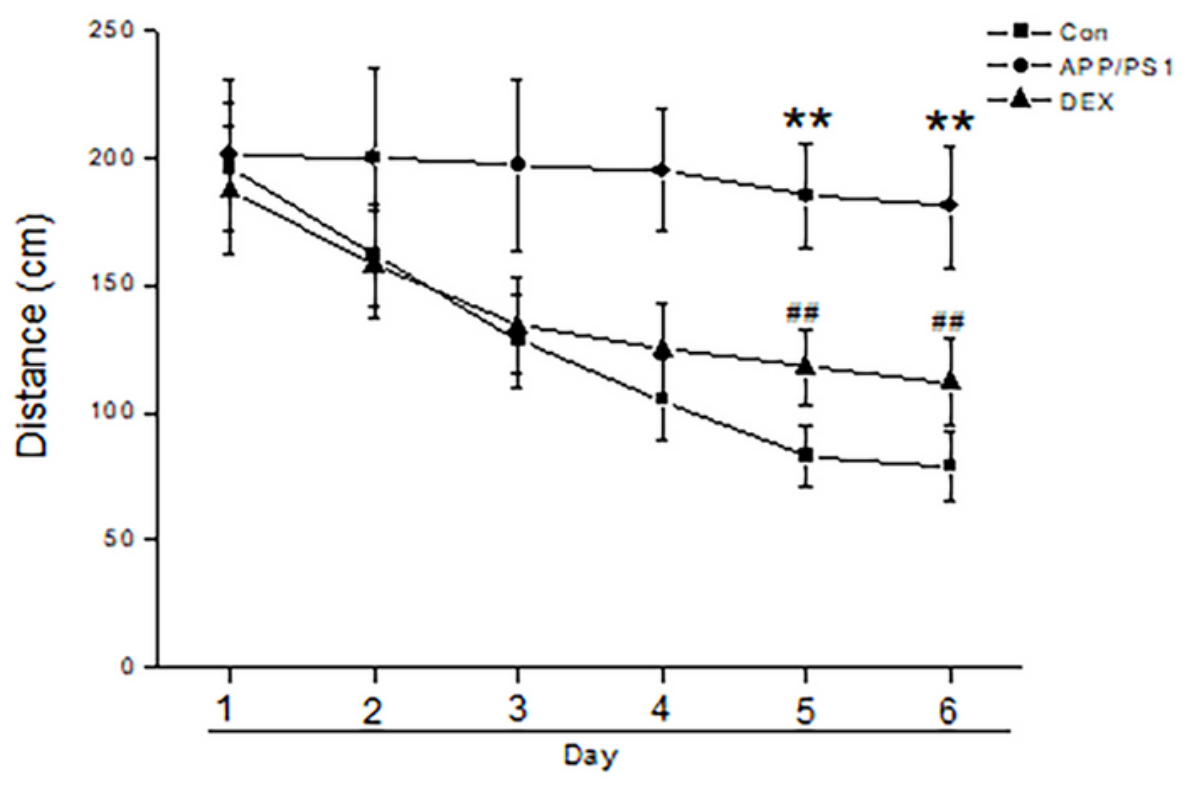

B

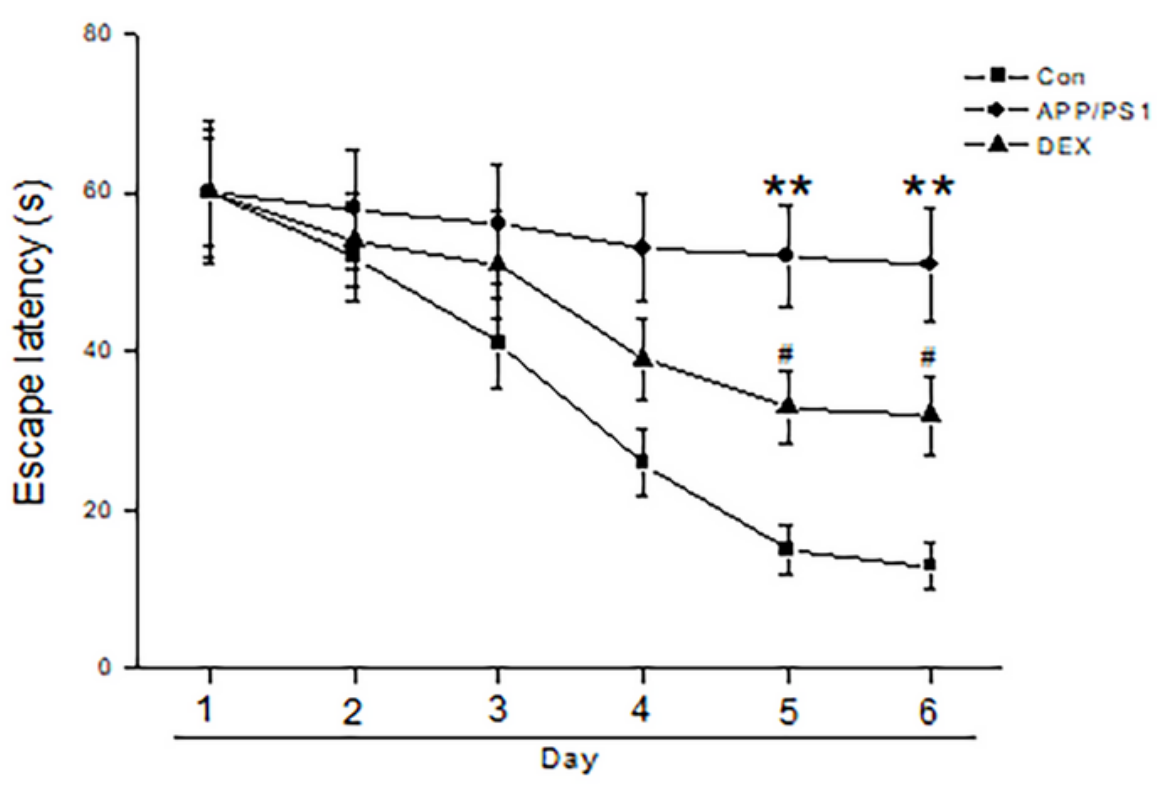

\section{Figure 1}

Examination of cognition using Water maze test. DEX prevents the decrement in distance $(A)$ and escape latency time (B) in Water Maze in APP/PS1 mice. APP/PS1 mice were administered DEX at the dose of $20 \mathrm{ug} / \mathrm{kg}$ by intraperitoneal injection for 28 days. Data are expressed as means \pm SD. $* * p<0.01$ vs Con. $\# p<0.05 ; \#$; $<0.01$ vs APP/PS1.n=10. 
Fig2

A

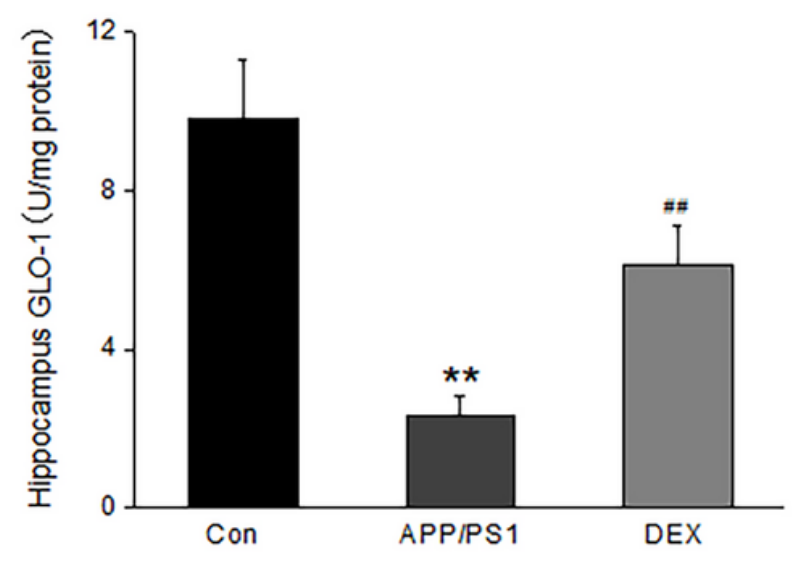

C

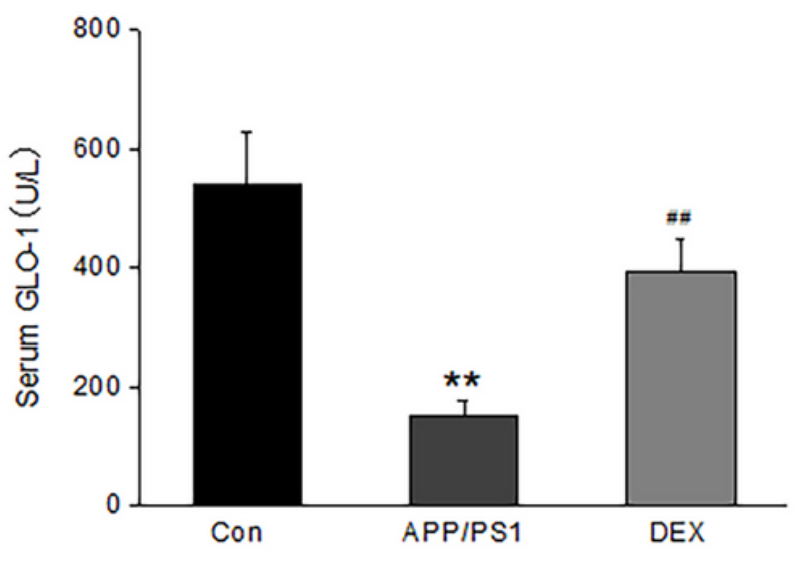

B

Con APP/PS1 DEX

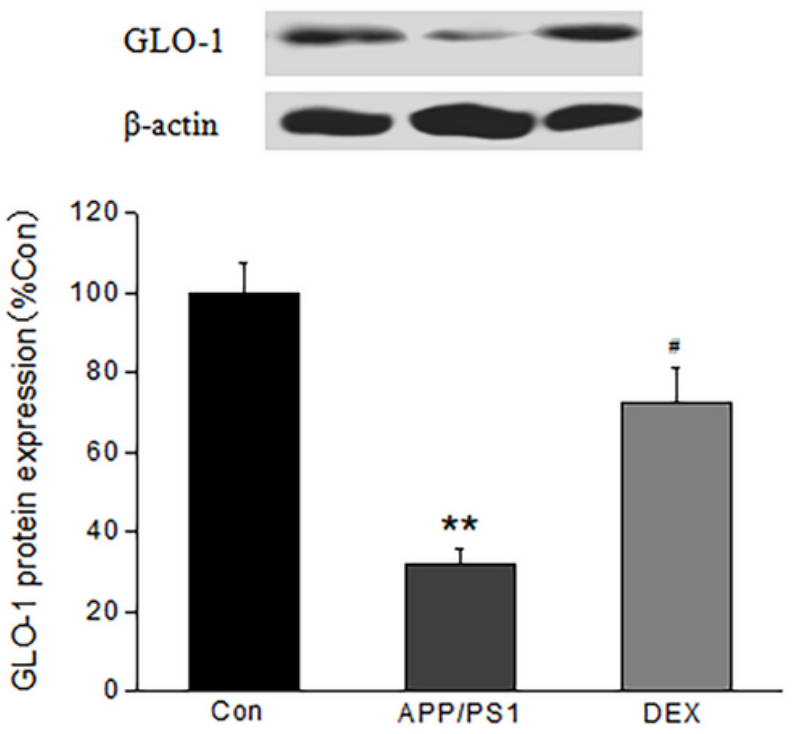

D

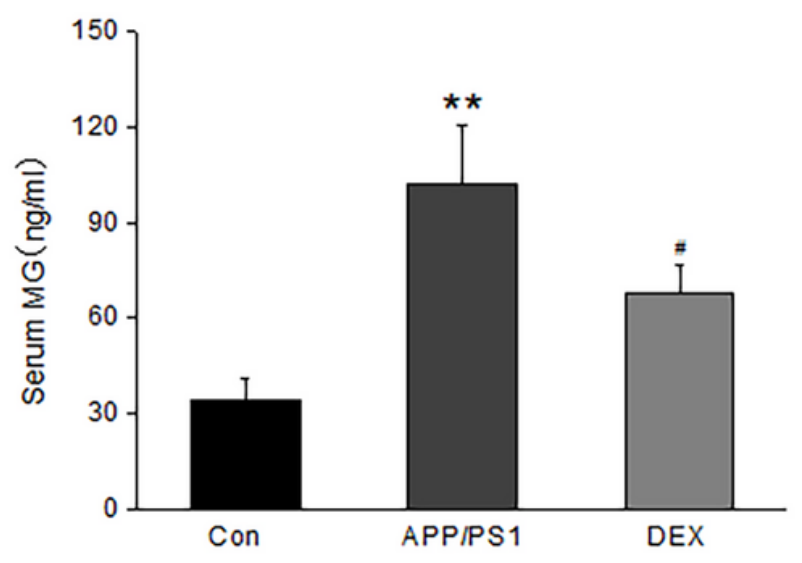

Figure 2

Western blot and ELISA analyses of Glo-1 and MG activity measurements in hippocampus and serum in APP/PS1 mice after 28 days DEX treatment. DEX increased the Glo1 level and decreased the MG level in APP/PS1 mice. (A) The activity of Glo1 in hippocampus homogenate determined by ELISA. (B) The expression of Glo1 in hippocampus determined by western blot. (C) The activity of Glo1 in serum determined by ELISA. (D) The activity of MG in serum determined by ELISA. $* p<005, * * p<0.01$ vs Con. $\# p<0.05 ; \# \#<0.01$ vs APP/PS1. $n=10$. 
Fig3

A

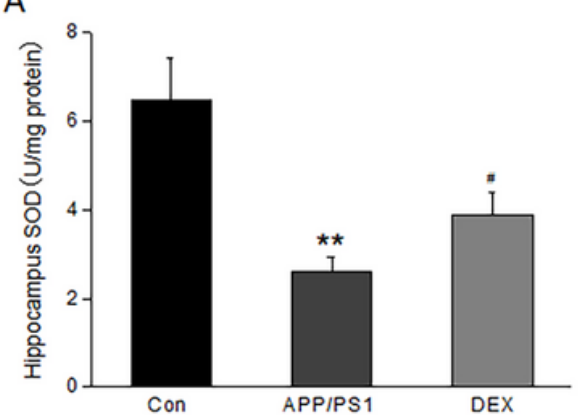

D

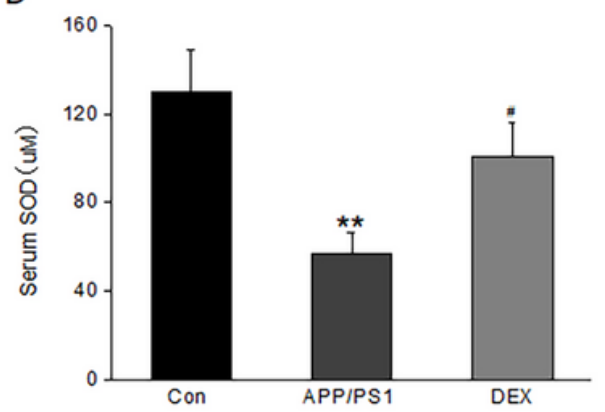

B

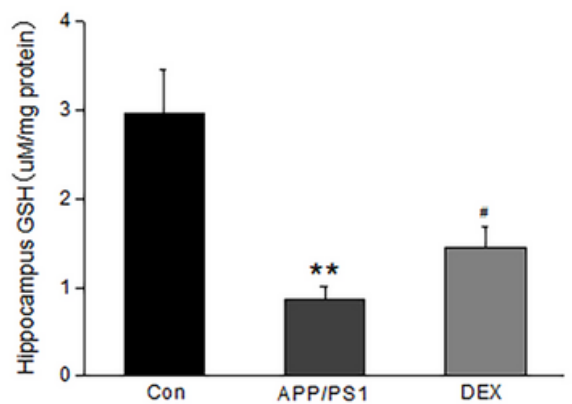

E

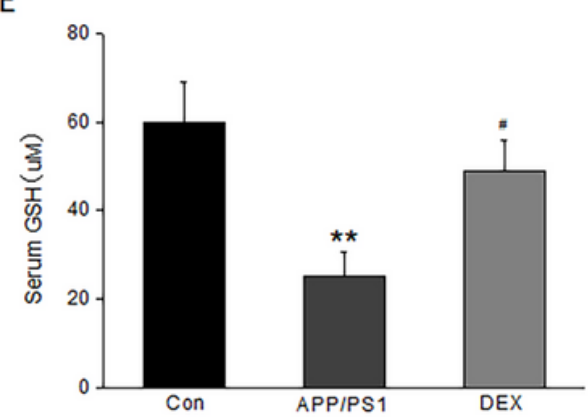

C

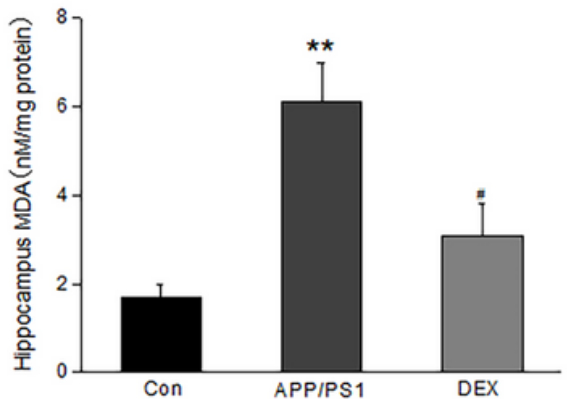

F

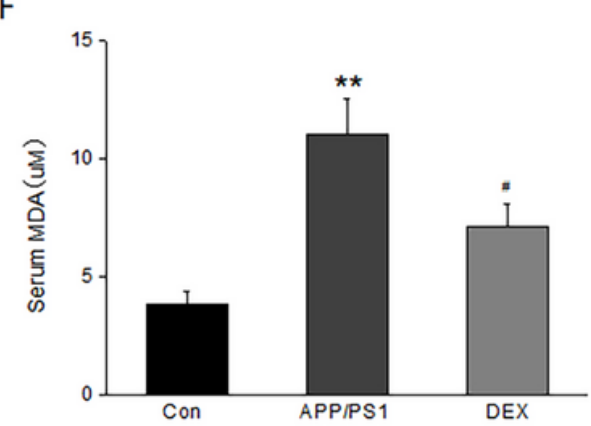

Figure 3

Examination of $\operatorname{SOD}(A, D), G S H(B, E)$, and $M D A(C, F)$ level in hippocampus and serum in APP/PS1 mice. APP/PS1 mice showed marked decrease in the activity of SOD and GSH with following by increase in the activity of MDA, which were reversed with DEX treatment. $* p<005, * * p<0.01$ vs Con. \#p<0.05; \#\#p< 0.01 vs APP/PS1. $n=10$. 
Fig4

A

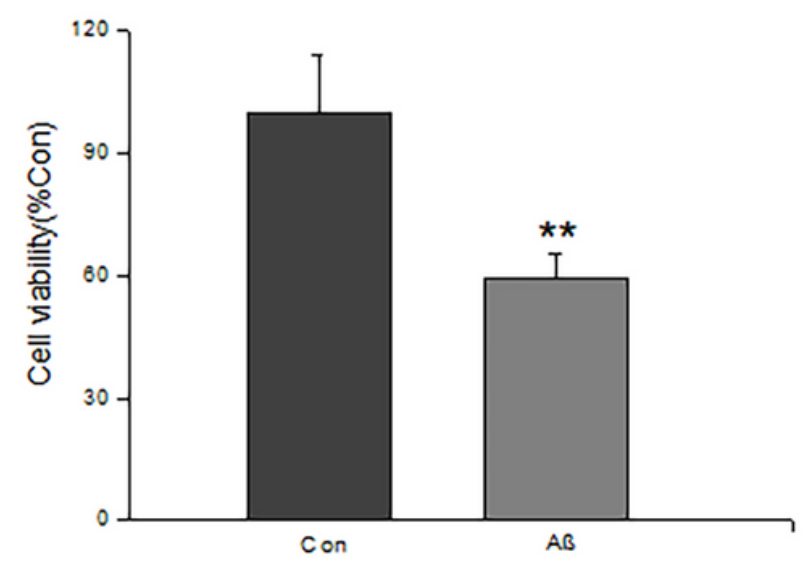

C

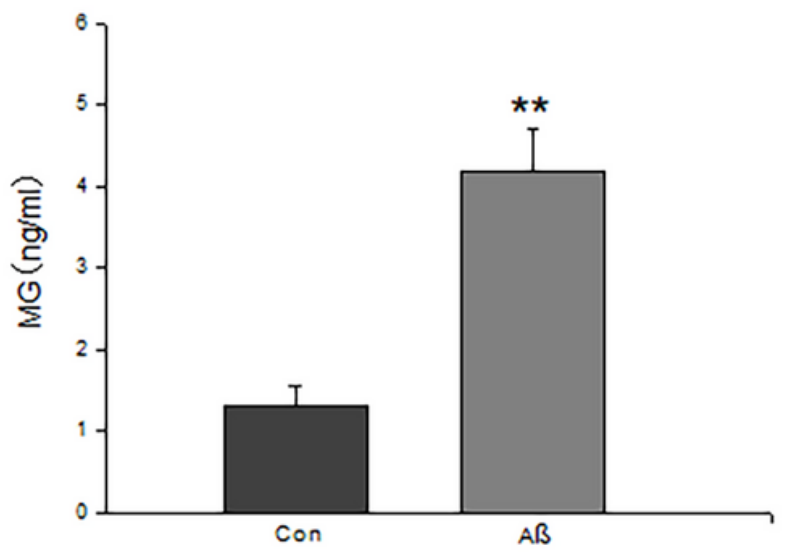

B

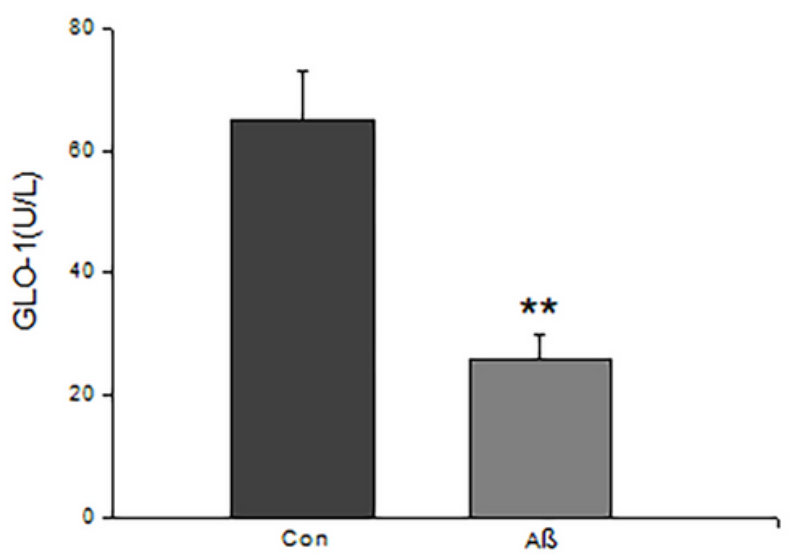

D

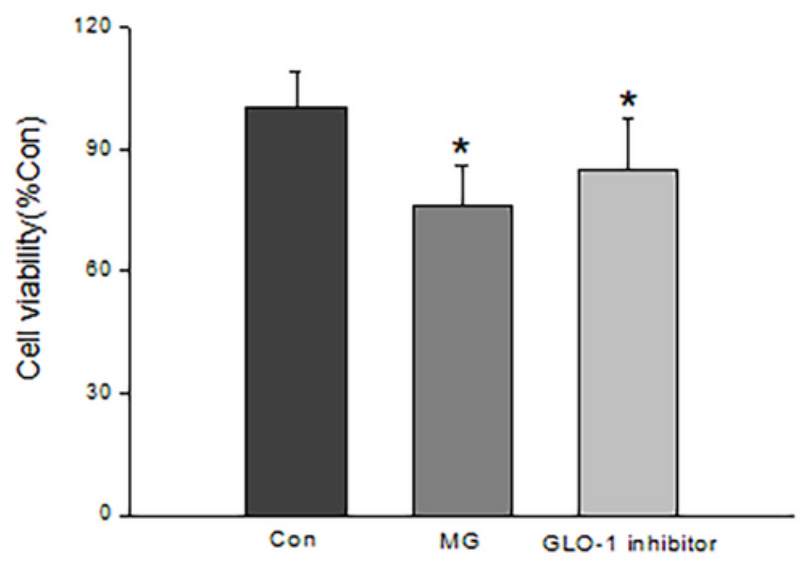

Figure 4

Identification of GLO-1 participating in A $\beta$-induced SHSY5Y cell viability decline. (A) A $\beta$ treatment induced SHSY5Y cell viability decline. The cell viability was determined by MTT assay. (B) The activity of Glo1 in SHSY5Y cell. (C) The activity of MG in SHSY5Y cell. (D) MG and GLO-1inhibitor caused cell viability decline. $* p<005, * * p<0.01$ vs Con. $\# p<0.05 ; \# \#<0.01$ vs $A \beta . n=6$. 
Fig5

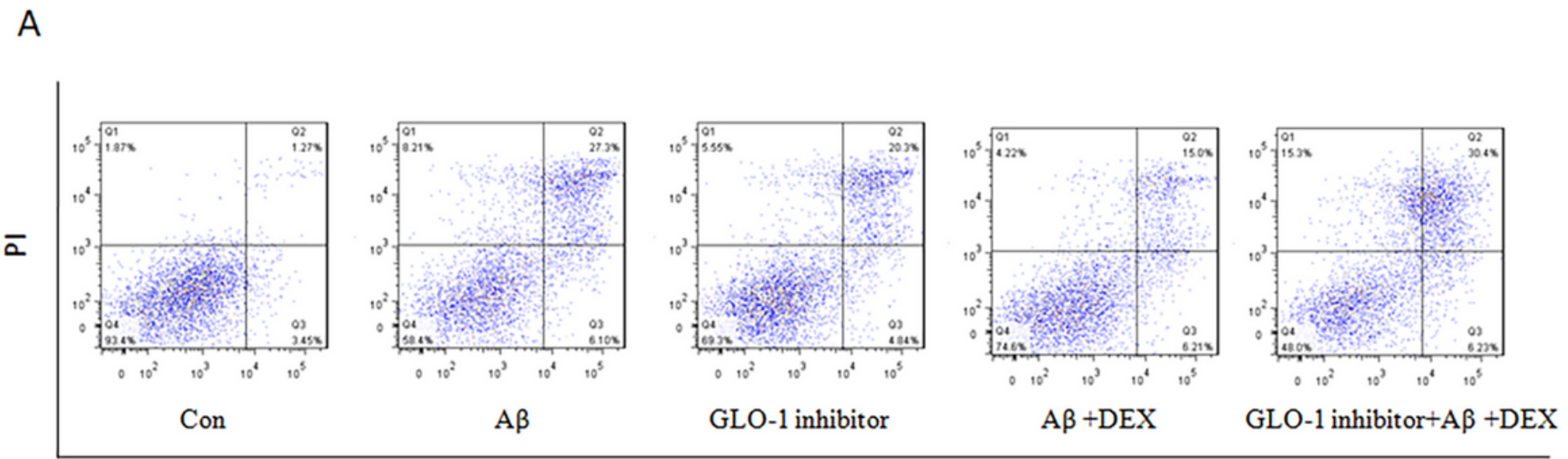

Annexin-v

B

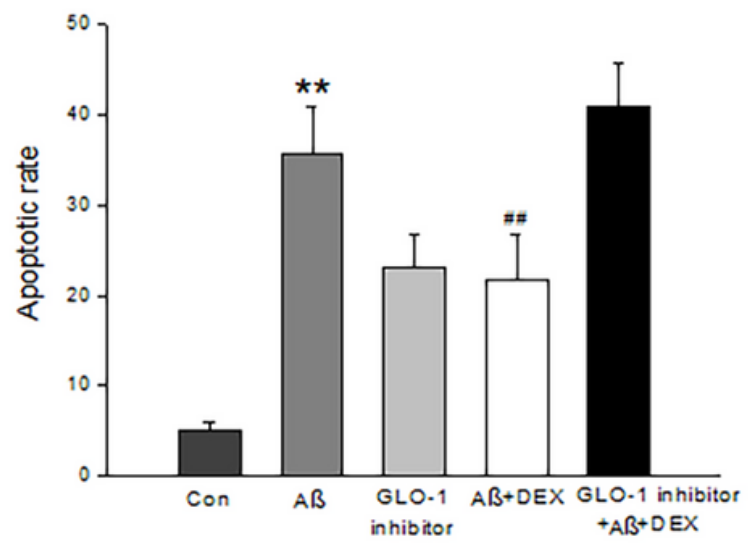

C

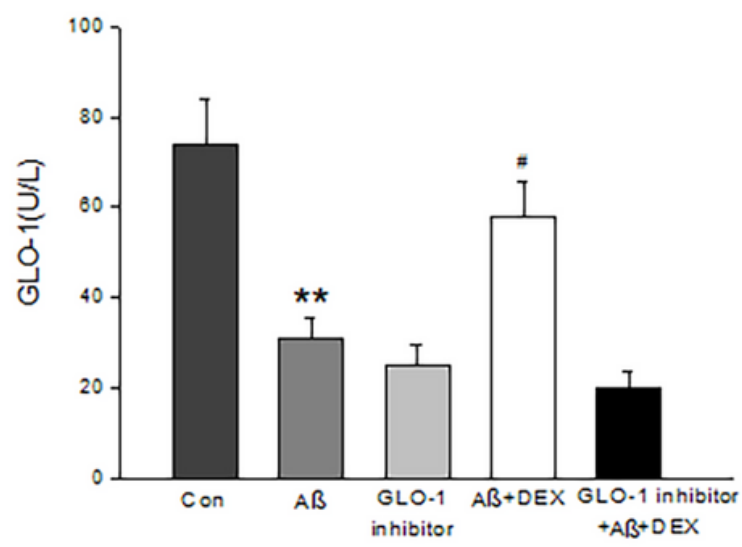

Figure 5

Electronic gating strategy for flow cytometric analysis of cell apoptosis. DEX reduced the apoptosis induced by $A \beta$. GLO-1 inhibitor could block the protective role of DEX $* p<0.05 ; * * p<0.01$ versus Con. $\# p<0.05 ; \# \# p<0.01$ versus $A \beta . n=6$. 
Fig6

A

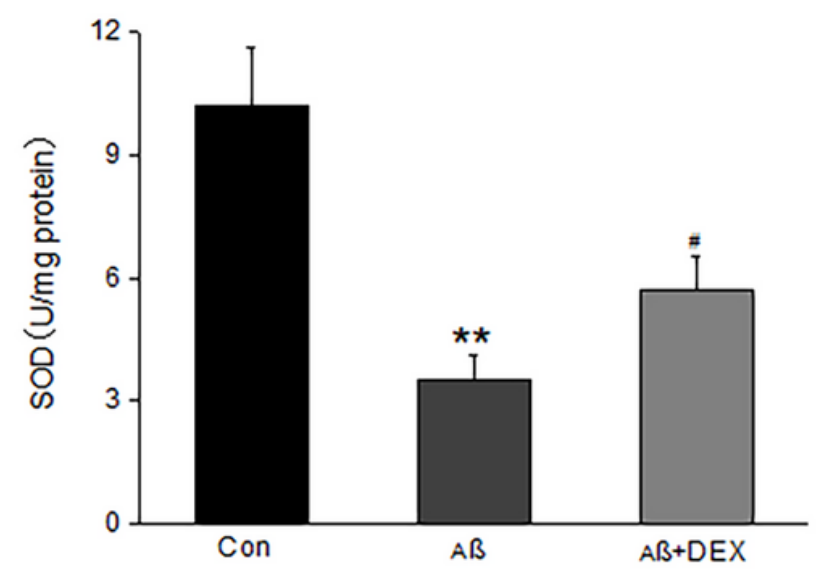

B

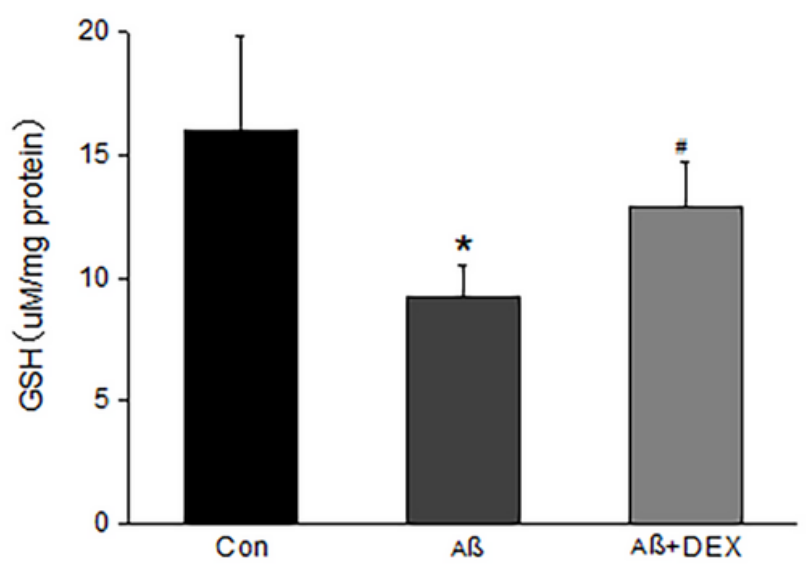

C

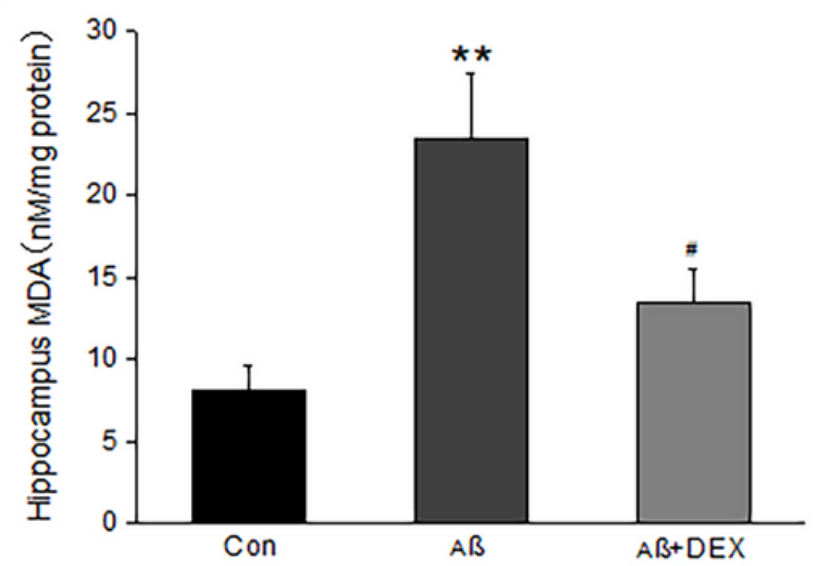

Figure 6

The activity of $\operatorname{SOD}(\mathrm{A}), \mathrm{GSH}(\mathrm{B})$ and $\mathrm{MDA}(\mathrm{C})$ in SHSY5Y cells. A $\mathrm{B}$ decreased the activity of SOD,GSH and increased the activity of MDA. DEX could reverse these damages. $* p<0.05 ; * * p<0.01$ versus Con. \#p $<$ $0.05 ; \# \# p<0.01$ versus $A \beta . n=8$. 